\title{
Auteur et image d'auteur en analyse du discours
}

Author and Image of the Author in Discourse Analysis

\section{Dominique Maingueneau}

\section{(2) OpenEdition}

Journals

\section{Édition électronique}

URL : http://journals.openedition.org/aad/660

DOI : $10.4000 /$ aad. 660

ISSN : 1565-8961

Éditeur

Université de Tel-Aviv

\section{Référence électronique}

Dominique Maingueneau, «Auteur et image d'auteur en analyse du discours », Argumentation et Analyse du Discours [En ligne], 3 | 2009, mis en ligne le 15 octobre 2009, consulté le 23 septembre 2019. URL : http://journals.openedition.org/aad/660 ; DOI : 10.4000/aad.660

Ce document a été généré automatiquement le 23 septembre 2019.

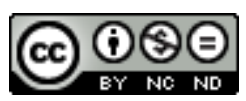

Argumentation \& analyse du discours est mis à disposition selon les termes de la licence Creative Commons Attribution - Pas d'Utilisation Commerciale - Pas de Modification 4.0 International. 


\title{
Auteur et image d'auteur en analyse du discours
}

\author{
Author and Image of the Author in Discourse Analysis
}

Dominique Maingueneau

1 Quand on veut étudier la notion d'« image d'auteur ", on se heurte a priori à trois difficultés :

- Comme le souligne la syntaxe, cette notion mobilise non pas une mais deux problématiques : celle de l'auteur, et celle de l'image d'auteur. La solution de facilité consiste à se focaliser sur l'image, en prenant l'auteur pour une donnée stable. Malheureusement, il n'en est rien : on est bien obligé de se demander de quelle instance l'image d'auteur est supposée être « l'image"

- Si la notion d'auteur est établie depuis longtemps dans le vocabulaire de la théorie littéraire ${ }^{1}$ et a fait l'objet d'intenses réflexions, celle d'image d'auteur est récente. Certes, on n'a pas manqué d'employer çà et là l'expression « image d'auteur », quoique plutôt sous la forme "l'image de tel auteur dans tel lieu et/ou à telle époque », mais ces études étaient menées du point de vue de la réception. Les questions que l'on peut poser aujourd'hui à partir de la notion d'image d'auteur étaient auparavant dispersées dans diverses rubriques de l'histoire littéraire et n'étaient pas intégrées dans un cadre théorique cohérent.

- La problématique de l'image d'auteur, mais aussi et surtout celle de l'auteur, se sont développées presque exclusivement sur des corpus littéraires. Il n'est donc pas évident d'en traiter dans une perspective plus large d'analyse du discours.

2 Autant dire que le texte qui va suivre ne prétend pas développer une théorie constituée, mais mettre en place un certain nombre de repères.

\section{Un tournant dans les études littéraires}

3 Le fait que la problématique de "l'image d'auteur" soit récente dans les études littéraires peut s'expliquer. On peut y voir une manifestation parmi beaucoup d'autres 
d'un tournant qui est en train de s'opérer autour des courants pragmatiques et de l'analyse du discours. Comme j'ai essayé de le montrer à diverses reprises (Maingueneau 2004; 2006),ce tournant implique une mise en cause la coupure immémoriale entre le "Texte", nécessairement majuscule, et son "contexte", coupure qui fondait tacitement la séparation entre "histoire littéraire » et étude immanente des œuvres, que celle-ci soit thématique, stylistique, narratologique... Cette coupure a été radicalisée à partir du 19e siècle dans le régime esthétique qui trouvé son couronnement dans le Contre Sainte-Beuve de Proust, qui établit une opposition quasi sacrée entre «moi créateur » et « moi social ». Une telle esthétique ne peut que rejeter une problématique de l'» l'image d'auteur ».

Cette coupure a su perdurer sans difficulté dans les approches de type énonciatif. Il suffit d'observer l'obstination avec laquelle on s'attache communément à bien séparer le « narrateur » et « l'écrivain », l'intratextuel et l'extratextuel. Elle peut même passer à l'intérieur de la notion d'auteur. On le voit dans ces quelques lignes extraites d'un document présenté par le futur Pape, le Cardinal Ratzinger, au nom de la « Commission biblique pontificale » qui synthétise et vulgarise diverses approches de la Bible; dans la rubrique « analyse narrative » on trouve ces lignes :

Plusieurs méthodes introduisent une distinction entre « auteur réel» et «auteur implicite », « lecteur réel » et « lecteur implicite ». L'» auteur réel » est la personne qui a composé le récit. Par "auteur implicite » on désigne l'image d'auteur que le texte engendre progressivement au cours de la lecture (avec sa culture, son tempérament, ses tendances, sa foi, etc.)

[http://www.portstnicolas.org/Interpretation-de-la-Bible-dans-l.html, accédé le $8 / 12 / 2008] .^{2}$

Une telle distinction est indiscutable, mais on voit que, prise d'une certaine façon, elle conforte la topique immémoriale d'un intérieur et d'un extérieur du texte. En témoigne l'interprétation de la notion d' » image d'auteur », qui est enfermée dans l'espace du texte. Les choses ne seraient sans doute pas si simples si l'on s'interrogeait sur l'auteur en tant qu'instance qui signe le texte : dans ce cas, en effet, il ne s'agit ni de la personne de l'» auteur réel » ni de l'» auteur implicite ».

6 En fait, les notions d'auteur et d'image d'auteur excèdent les catégorisations traditionnelles. L'» auteur " - celui dont traite Foucault dans son fameux texte de 1969 - n'est ni l'énonciateur, corrélat du texte, ni la personne en chair et en os, qui serait renvoyée au "contexte». Quant à "l'image d'auteur », elle n'appartient ni au producteur $^{3}$ du texte ni au public ; elle constitue une réalité instable, le produit d'une interaction entre des intervenants hétérogènes.

7 Si l'on peut développer aujourd'hui une réflexion sur l'image d'auteur, c'est que la mise en scène discursive de l'écrivain n'est plus appréhendée comme un ensemble d'activités qui demeureraient à l'extérieur de l'enceinte sacrée du Texte, mais comme une dimension à part entière à la fois de la communication littéraire comme co-énonciation et du discours littéraire comme activité dans un espace social déterminé. On retrouve à un niveau de complexité supérieur le principe même d'une "scène d'énonciation » des œuvres (Maingueneau 1993, 2004): énoncer en littérature, ce n'est pas seulement configurer un monde fictionnel, c'est aussi configurer la scène de parole qui est à la fois la condition et le produit de cette parole. Des problématiques étroitement liées comme celles de «l'ethos» (Maingueneau 1987, Amossy (éd.) 1999), de la "posture » (Viala 1993, Meizoz 2002) ou, plus récemment, de "l'image d'auteur", chacune dans leur ordre propre, vont dans le même sens. 
8 La difficulté consiste évidemment à ne pas passer d'un «textualisme » à un «sociologisme» qui nous ramènerait, sous un visage différent, au dispositif traditionnel.

\section{En analyse du discours}

9 En matière de réflexion sur l'auteur l'analyse du discours souffre d'un déficit certain. Dans leur grande majorité, ceux qui s'en réclament ont éludé la fameuse question posée par Michel Foucault à la fin des années 1960 («Qu'est-ce qu'un auteur?»). Pourtant, une analyse du discours qui assume pleinement la diversité des genres de discours ne devrait pas éluder cette question, qu'elle rencontre sans cesse.

10 La réticence des analystes du discours peut se comprendre, si l'on considère les conditions dans lesquelles s'est développé leur champ de recherche.

11 Je n'insisterai pas sur les recherches menées en Amérique du Nord, où ce sont de manière privilégiée les corpus conversationnels qui ont été pris en compte; pour ce type de corpus l'analyste peut avoir l'impression que la question de l'auctorialité est dépourvue d'intérêt: les locuteurs sont là, en chair et en os, les paroles qui sortent de leur bouche sont leurs paroles. Ici l'auteur s'absorbe dans le locuteur.

12 En revanche, le déficit de réflexion sur l'auctorialité peut sembler plus surprenant dans l'analyse du discours européenne, et surtout francophone, où l'on accorde une grande importance aux corpus écrits.

13 On pourrait expliquer cette réticence par le fait que l'analyse du discours, dans la mesure où elle a occupé les corpus laissés libres par les facultés de lettres traditionnelles, a largement évité aborder des types de discours comme le discours littéraire, religieux ou philosophique, domaines où la question de l'auctorialité est difficilement éludable.

Mais il est aussi permis de se demander si la problématique de la polyphonie linguistique, et plus largement tout ce qui tourne autour de l'hétérogénéité ou de la modalisation, n'a pas fait obstacle à une réflexion sur l'auctorialité. Ces travaux posent en effet la question de la pluralité des sources énonciatives, mais en demeurant dans un espace linguistique. Ce faisant, ils se placent dans le prolongement de certains présupposés de la linguistique moderne, qui préfère l'oralité et souscrit spontanément à la méfiance que Socrate exprimait dans le Phèdre à l'égard de l'écriture : un écrit n'a pas de père pour l'assister. De fait, par nature un écrit a un auteur, pas un locuteur. L'auctorialité vient miner la transparence du langage, elle excède la stricte communication linguistique.

\section{Auteur et image d'auteur}

La notion d'auteur se construit sur deux restrictions successives. La première ne retient parmi les emplois du mot » auteur » que ceux qui ont trait à la production d'un texte. La notion d'auteur n'est en effet nullement réservée aux productions verbales. On ne dira pas, sauf dans des contextes très particuliers, "l'auteur de la voiture " ou "l'auteur du bricolage», mais on voit proliférer dans les médias des désignateurs tels que «l'auteur des injures", "l'auteur des coups de couteau», "l'auteur de l'agression », etc., qui relèvent clairement de la sphère judiciaire. Cette catégorisation 
judiciaire peut concerner tout aussi bien des énoncés, comme on le voit dans ces deux exemples pris au hasard sur Internet :

Titre sur Internet :

- «Ligue 1 : l'auteur des propos racistes sera jugé le 18 mars.»

[http://www.europe1.fr/Sport/Football/L1-L2, consulté le 31/10/08]

- «Lors du procès devant les assises des Alpes-Maritimes, au mois de février 1994,

les graphologues Florence Buisson-Debas et Gilles Gessner, assermentés auprès du

tribunal de Nice, ont affirmé que Ghislaine Marchal était bien l'auteur de la phrase

fatale. »

[http://hebdo.nouvelobs.com/hebdo/parution/, consulté le 31/10/08]

Ici «la phrase fatale» ou "les propos racistes» ne sont pas envisagés comme des entités linguistiques seulement mais au même titre que des coups de couteau ou un vol : il s'agit d'imputer une responsabilité dans un cadre judiciaire.

Mais la notion d'auteur qui nous concerne ici n'est pas à proprement parler d'ordre judiciaire - même si elle renvoie elle aussi à une origine et une responsabilité -, mais d'ordre textuel. Le problème est alors de savoir à quelles conditions un texte est susceptible d'avoir un "auteur». A l'évidence, on dira difficilement qu'une conversation a des « auteurs » : on préférera parler de "participants ». Une production textuelle, semble-t-il, n'est « auctorisable » que si elle fait l'objet d'une re-présentation qui permet de l'appréhender de l'extérieur, comme un tout. Ce qui n'est pas le cas dans un échange verbal en cours. Il y a " auctorisation » si l'énoncé, fût-il oral, est délimité et introduit dans un espace où il $\mathrm{y}$ a nécessité de déterminer une attribution parmi un ensemble d'origines possibles, une responsabilité. Dans ce terme se mêlent intimement assignation d'origine ( $\mathrm{X}$ est la cause de l'énoncé) et dimension éthique ( $\mathrm{X}$ doit pouvoir «en répondre »).

La seconde restriction fait passer le terme " auteur » du fonctionnement relationnel au fonctionnement référentiel. La problématique de «l'image d'auteur » ne vaut que pour le second. Le fonctionnement «relationnel» institue en repère le texte produit: l'auteur de ce tract, de cette lettre, de ce manuel, de cet article... En revanche, le fonctionnement «référentiel » autonomise syntaxiquement l'auteur: "l'auteur d'un tract publicitaire " n'est pas, sauf situation exceptionnelle, "un auteur", pas plus qu'un percepteur qui écrirait beaucoup de lettres aux contribuables qui relèvent de sa compétence. Le Trésor de la Langue Française met en évidence cette restriction, qu'il aggrave même, en restreignant les domaines pour lesquels l'auteur s'autonomise :

[Sans compl. de n., absol. ou suivi d'un adj. déterminant l'orig., le genre, la qualité de l'auteur]

1. LITTÉRATURE

a) Celui ou celle dont la profession est d'écrire des romans, des pièces de théâtre, des œuvres d'imagination en vers ou en prose. Synon. écrivain, romancier, dramaturge.

19 Ainsi, alors qu'il n'y a aucune difficulté à dire par exemple qu'une "image d'auteur » est attachée à quelqu'un qui écrit des ouvrages religieux, politiques, philosophiques..., il semble, comme le montre le TLF, que le fonctionnement référentiel soit plutôt focalisé sur les producteurs de littérature. On notera cependant que, dans l'usage, " auteur» et "image d'auteur" s'appliquent aussi à diverses sortes de producteurs esthétiques : photographes, cinéastes en particulier (on parle ainsi de "film d'auteur ») ... Tout se passe donc comme si «l'auteur» et "l'image d'auteur" tendaient à se spécialiser dans la sphère esthétique. 
Pour autant, il reste à déterminer où passe la frontière entre les deux fonctionnements, à partir de quand «l'auteur de X » devient «un auteur» tout court. On est tenté d'invoquer un critère comme la qualité, comme semble le faire G. Leclerc :

Pour être considéré comme un auteur, le sujet énonciateur doit avoir donné à ses paroles, à son texte, une marque propre qui les distingue des énoncés courants, des propos de la vie quotidienne. L'œuvre textuelle est un énoncé "original», innovationnel, qui, à la différence des poncifs, des clichés, des stéréotypes, des idées reçues, renferme une «idée neuve », inédite, jamais dite dans la culture (1998: 50-51).

21 Mais on ne voit pas pourquoi un producteur peu original ne serait pas un auteur. En fait, il semble bien qu'ici deux acceptions d'« auteur » interfèrent. L'une réfère plutôt à un statut social ; un homme politique, par exemple Valéry Giscard d'Estaing ${ }^{4}$, a beau avoir publié au cours de sa carrière quelques ouvrages politique et autobiographiques, voire un roman ${ }^{5}$, on le catégorisera difficilement comme " auteur ", sans complément du nom. L'autre acception implique une évaluation, indépendamment de tout statut social : elle ne vise que le "véritable » auteur, dont la forme accomplie est le "grand auteur ", susceptible de figurer dans une anthologie à l'usage des établissements scolaires.

Ce que le producteur de littérature a de singulier c'est qu'il prétend se qualifier par la seule production de textes. En revanche, un homme politique, un prêtre ou un savant ne sont pas censés faire de la production de textes l'essentiel de leur activité. Il suffit d'ailleurs de considérer un discours voisin, le discours philosophique, pour voir que sous certains aspects il diffère sur ce point du discours littéraire, alors même que nul ne contesterait au philosophe une image d'auteur. En particulier, la relation entre «l'homme » et «l'œuvre» y apparaît différente. En témoigne le fait que le discours philosophique est réticent à l'égard de la pseudonymie, de la mystification, bref de tous les jeux sur l'auctorialité auxquels se livrent les écrivains. En outre, les écrivains ont des existences souvent chaotiques, souvent sans rapport évident (en fait, c'est tout l'art des analystes de montrer la cohérence cachée) avec leur œuvre. A ces deux traits les auteurs philosophiques ne souscrivent pas: en règle générale leurs textes ont un répondant qui renvoie à leur personne, pas un pseudonyme ${ }^{6}$, un responsable devant l'humanité, et leur vie ne cesse d'essayer de se mettre en conformité avec les règles qui régissent leur univers de pensée. Le comble est atteint quand, comme dans la philosophie antique, la notion de doctrine ne distingue pas entre doctrine philosophique et art de vivre. Les biographies des philosophes attirent donc moins que celles des écrivains. Cela se vérifie aussi au niveau de l'exégèse des œuvres: la démarche qui consiste à mettre en relation les événements de la vie personnelle et les fictions (Du Bellay déçu par Rome et écrivant Les regrets, Molière jaloux d'Armande Béjart, Lamartine écrivant "Le lac» après la mort de la femme aimée...), est périphérique en philosophie. Les spécialistes se plaisent d'ailleurs à croire qu'il n'est nul besoin de s'intéresser à la vie des philosophes. Peu de spécialistes de littérature diraient de leur écrivain préféré ce que dit G. G. Granger au début d'un livre consacré à Wittgenstein :

Le personnage d'un philosophe n'est certainement pas ce qui importe, et je laisserais volontiers de côté toute anecdote, n'était la curiosité invincible que chacun nourrit à l'égard des détails concrets touchant le caractère et la vie de ceux dont il admire les œuvres $(1990: 17)^{7}$. 


\section{Les trois dimensions de la notion d'auteur}

En fait, si l'on parle si volontiers et sans s'interroger davantage de l'» œuvre » d'un auteur, c'est qu'on la suppose définie par une certaine fonction d'expression. On admet qu'il doit y avoir un niveau (aussi profond qu'il est nécessaire de l'imaginer) auquel l'œuvre se révèle, en tous ses fragments, même les plus minuscules et les plus inessentiels, comme l'expression de la pensée, ou de l'expérience, ou de l'imagination, ou de l'inconscient de l'auteur, ou encore des déterminations historiques dans lesquelles il était pris » (1969b : 35).

On notera que la notion d'image d'auteur va dans un sens opposé, puisqu'au lieu de replier l'unité d'un ensemble de textes sur la profondeur cachée d'une expression, elle déploie un espace de relations : relations à son texte de l'auteur, qui prend en compte les représentations des publics, représentations de cet auteur et de ses textes dans ces publics.

L'activité proprement littéraire se distingue d'autres vouées également à la production de textes, comme le journalisme ou la politique, en cela que toute personne qui publie un texte qui relève de la sphère esthétique devient ipso facto " auteur-auctor » en puissance. Mais il ne sera pleinement auctor, source d'autorité, que si des tiers lui 
construisent une « image d'auteur » qu'il peut gérer. Dès lors, on comprend la stratégie de nombreux écrivains des $\mathrm{XVII}^{\mathrm{e}}$ ou XVIII ${ }^{\mathrm{e}}$ siècles qui dans leur préface font mine de ne pas être l'auteur de leur texte, d'être eux-mêmes ce tiers qui accrédite le texte (Herman, Kozul, Kremer 2008). faudrait que ces textes deviennent les composants d'un Opus. Or la plupart des textes d'un homme politique, surtout quand ils sont au gouvernement, relèvent de routines et ne sont que l'expression d'une politique définie ailleurs. Il y a néanmoins des circonstances où l'homme politique est censé s'exprimer en son nom, au sens fort. Cela ne fait aucun doute, par exemple, pour l'Appel du 18 juin du général de Gaulle. Le caractère singulier d'un événement de parole augmente évidemment ses chances d'appartenir à un Opus. Dans ces conditions, les vœux adressés à la nation chaque Premier de l'an par le Président de la République ne relèvent pas a priori d'un Opus; mais une décision éditoriale, en particulier si on veut publier les » œuvres complètes » du Président, peut toujours convertir ces vœux en composants d'un Opus ${ }^{10}$.

(3) Il faut ensuite distinguer la situation de l'» auctor » en puissance, qui est identifiable mais qui passe inaperçu, et celle de 1 » auctor » identifié, le seul qui aura une « image » puisque le seul qui entre en interaction avec d'autres instances que lui-même. Un roman, un essai, un livre d'histoire dont personne ne parle ne font pas accéder réellement l'auteur à une image. Mais tout est question de degré ; sur ce point la nature des tiers impliqués joue un rôle essentiel. Un recueil de poésie publié à compte d'auteur et dont ne parle que l'entourage immédiat du producteur lui confère une qualité d'« auctor » extrêmement faible; en revanche, un essai qui est publié par un grand éditeur, recensé par divers magazines et qui conduit son auteur dans un studio de télévision confère un fort coefficient d'« auctorité ».

A la limite, on peut concevoir qu'un "auctor" prestigieux intègre à son Opus un ensemble de textes d'autres «auctores », pour peu que cet ensemble exprime un point 
de vue personnel. C'est le cas par exemple s'il publie une anthologie des «cent plus beaux poèmes de la littérature française ». Le caractère prépondérant du point de vue personnel de celui qui a sélectionné les textes l'emporte ici largement sur le fait que ces textes ne sont pas de lui. Ce type d'anthologie est très différent d'une anthologie destinée à l'enseignement secondaire, dans laquelle les auteurs du manuel doivent se présenter comme les médiateurs d'une institution extrêmement contraignante. Dans ce cas, il leur est pratiquement impossible d'accéder au statut d'« auctor ».

(4) Certains auteurs accèdent même au statut d'auctor majeur: leur figure est si saillante que l'on publie des textes d'eux qui n'étaient pas destinés à être publiés : brouillons, correspondance privée, devoirs d'écolier, carnets, journaux... Foucault avait souligné ce point :

le nom «Mallarmé » ne se réfère pas de la même façon aux thèmes anglais, aux traductions d'Edgar Poe, aux poèmes, ou aux réponses à des enquêtes; de même, ce n'est pas le même rapport qui existe entre le nom de Nietzsche d'une part et d'autre part les autobiographies de jeunesse, les dissertations scolaires, les articles philologiques, Zarathoustra, Ecce homo, les lettres, les dernières cartes postales signées par "Dionysos" ou "Kaiser Nietzsche", les innombrables carnets où s'enchevêtrent les notes de blanchisserie et les projets d'aphorismes. (1969b : 35)

Certains des genres que cite ici Foucault sont des œuvres au sens fort (poèmes pour Mallarmé, Zarathoustra, Ecce homo pour Nietzsche), ou des œuvres inabouties (autobiographies de jeunesse); d'autres sont des textes qui contribuent au travail de « réglage» ou de "figuration» du producteur (Maingueneau 2004): traductions, réponses à des enquêtes, articles philologiques ; d'autres enfin n'ont pas été conçus par le producteur comme destinés à sortir de la sphère privée: carnets, dissertations, thèmes anglais. Pour ces « auctores " majeurs, ce sont des décisions éditoriales fondées sur des opérations interprétatives qui font que tel ou tel genre par nature exclu par le créateur de la publication, va contribuer à son image d'auteur.

Force est donc de prendre acte de la distorsion entre le foisonnement des formes d'auctorialité (tout genre de texte a un auteur-répondant et l'ethos correspondant) et l'extrême restriction des individus susceptibles d'être "auteurs-auctores", associés à une « image d'auteur ».

\section{Une « ontologie » auctoriale}

Une question que nous avons jusqu'ici laissé de côté est la nature des entités qui sont susceptibles d'être dites " auteurs ", au sens d' " auctores ». C'est là un problème délicat car de multiples paramètres doivent être pris en compte simultanément. Dans les pages qui suivent, je ne vais pas définir a priori quelles sont les conditions nécessaires et suffisantes pour occuper la position d'auteur (à supposer que de telles conditions existent), mais réfléchir à partir d'un certain nombre de « cas » d'instances auctoriales susceptibles d'accéder à la dignité d'auteur.

Une condition nécessaire - mais pas suffisante, évidemment - pour qu'il y ait " auctor » est l'existence d'un nom d'auteur. Foucault rappelle à ce propos que "l'œuvre », ce que nous appelons ici l'Opus, c'est communément « une somme de textes qui peuvent être dénotés par le signe d'un nom propre » (1969b : 34). A cela on peut ajouter que l'Opus doit être l'» expression » de son auteur. Prenons l'exemple des publicités produites par une marque commerciale; cette dernière se présente comme une entité constituée 
autour d'un nom propre et qui se pose en source et en garant d'un ensemble cohérent de textes censés exprimer sa "personnalité ». Pour autant, il apparaît difficile de dire qu'une marque est un auteur. Déjà, on notera que l'unité des textes dont la marque se pose en responsable est indépendante de l'identité des employés qui sont en charge de sa communication externe. De plus, indépendamment des difficultés soulevées par le fait qu'une marque n'est pas à proprement parler un humain, la dissociation entre la marque et le producteur effectif des textes, en l'occurrence l'agence de publicité, semble problématique (en fait, il peut s'agir de diverses agences, en fonction de contrats qui sont renégociés régulièrement). L'agence de publicité elle-même peut utiliser des employés différents tout en restant la même agence. Si la notion d'Opus implique tacitement l'existence d'une entité douée d'un "point de vue», d'une « conviction », d'un «style ", ce qui fait obstacle à ce qu'une marque soit un «auctor ", c'est le fait qu'elle ne soit pas arrimée à l'unité imaginaire d'une conscience et d'une histoire dont les textes seraient « l'expression ». En revanche, rien n'empêche tel(s) ou tel(s) individu(s) travaillant pour l'agence de publicité, voire l'agence, d'accéder à un statut d' » auctor ", dès lors qu'ils sont identifiables comme personnalités créatrices dans un espace de production symbolique.

41 A présent, qu'en est-il de l'homme politique qui n'écrit pas lui-même ses discours ? Comme la marque commerciale, il n'est pas le producteur de son texte. La différence est néanmoins nette entre les deux cas de figure. Le texte de l'homme politique aurait pu être écrit par lui, il exprime sa ligne politique et il le profère en personne ${ }^{11}$. En revanche, la publicité se caractérise par son éloignement des producteurs qu'elle fait travailler. Dans un domaine comparable, celui de la haute couture, on fait habituellement la distinction entre l'auteur, à savoir le créateur qui a dessiné la collection, et la marque pour laquelle il travaille : « Karl Lagerfeld pour Chanel ».

En fait, le cas de la publicité électorale nous montre que la notion de "point de vue ", voire de "conviction» ne suffit pas : il faut aussi une prise en charge directe. Une publicité qui fait la promotion d'un candidat à une élection fait de ce dernier non un locuteur mais une sorte de personnage, qui recourt éventuellement au discours direct. Aux USA, à la fin des spots de la campagne présidentielle on entend la voix du candidat qui dit «I am X (G. Bush, J. Kerry, B. Obama...) and I approve this message ». Ce faisant, le candidat affirme que ce spot exprime son point de vue, mais il ne se pose pas comme sa source, il n'en est pas le répondant.

Nous avons suggéré que, dans certaines conditions, une agence de publicité pouvait accéder au statut d'auteur. Mais cela n'est possible que si l'on donne un statut aux entités collectives. A cet égard, on peut penser que des groupes tels que les partis politiques ou les mouvements littéraires sont dans une situation plus propice.

Leurs textes, produits d'une élaboration collective négociée, sont au terme du processus attribués à un auteur indivisible. Plus précisément, il faut distinguer deux cas très différents: a) celui où les individus qui participent à l'élaboration appartiennent à une "communauté de conviction" forte ; b) celui où l'on cherche à aboutir à un compromis qui préserve la diversité des instances négociantes : ainsi les syndicalistes négocient-ils des motions de synthèse. Ces textes de compromis maintiennent une hétérogénéité, même s'ils sont assumés par une communauté à laquelle appartiennent les divers intervenants (le syndicat X, le parti Y). Dans un texte de "synthèse ", le lecteur expert est en effet capable de repérer les apports respectifs 
de chacun. Voici un document significatif, qui émane d'une section du " Nouveau Parti Socialiste "; nous avons mis en italique le passage qui nous intéresse ici.

27 novembre 2005 par NPS29

Chers camarades,

Il appartient à chacun d'entre vous de se faire librement et en connaissance de cause son jugement. C'est pourquoi, maintenant qu'il est définitif nous vous adressons ci-joint le texte intégral de la motion de synthèse issu de la Commission des résolutions. [à télécharger ci-après] Comme vous le savez, le NPS avait un mandat de négociation, voté par l'AG des délégués qui portait sur les quatre points majeurs que nous avions défendu tout au long des débats du Congrès : - L'Europe et la mondialisation - Les questions économiques et sociales - Les institutions et la démocratie - La rénovation

Dans le texte que nous vous adressons vous trouverez en fond grisé les amendements que nous avons fait inclure lors des six heures de discussion. En annexe nous avons collationné selon nos quatre grands thèmes les différents amendements pour vous permettre de mesurer l'ampleur et la cohérence des modifications obtenues par rapport aux orientations que nous avons défendu [sic] dans les débats du Congrès. Ce sont ces avancées qui nous ont conduit en conscience à voter à une très large majorité d'entre nous la synthèse.

Vous comprendrez aussi que nous ne souhaitons pas davantage entrer dans une polémique dont nous ne voyons pas qui elle sert, en tous cas certainement ni le NPS ni le PS.

Nous nous contenterons d'affirmer pour ce qui nous concerne que notre souci majeur est de préserver, dans le respect d'une démarche collective, qui pour avoir ses contraintes a aussi sa force et sa légitimité, notre unité et donc la continuité du NPS.

Bien des combats restent à poursuivre dans la durée, et nous avons besoin, pour les mener de rester unis, cohérents et respectueux entre nous.

Dans l'attente de vous revoir,

Amitiés socialistes.

Henri Emmanuelli, Vincent Peillon, Benoît Hamon, Pascal Cherki, Renaud Lagrave, Jean Guérard, Jean Louis Carrère, Pascal Terrasse, Gilbert Roger, Jacques Rigaudiat, Delphine Mayrargues, Régis Juanico, David Assouline, Isabelle Thomas, Michel Vergnier, Germinal Peiro, Isabelle Martin, Christian Martin, Philippe Darriulat, Josy Pouyeto, Membres de la Commission de résolution.

Site: «Pour un Nouveau Parti Socialiste en Finistère »[http://nps29.free.fr/ article.php3?id_article=105, consulté le 2 novembre 2008].

Cette lettre adressée aux militants évoque la motion de synthèse pour mieux souligner son hétérogénéité : le Nouveau Parti Socialiste en revendique certains passages qu'il porte à l'attention de ses membres.

En revanche, les communautés de conviction forte, où le texte émerge de la collaboration de points de vue convergents, impliquent la fiction d'un groupe indivisible, beaucoup plus propice au statut d'«auctor». Un parti politique, ou un courant bien identifié dans un parti, ne sont pas, en effet, des entités que l'on pourrait dire "compactes", comme par exemple le groupe de peintres regroupés à Munich autour de Kandinsky sous le nom «Der blaue Reiter $»^{12}$. Il s'agit d'un groupe très restreint, dont les membres étaient eux-mêmes des créateurs à part entière, associés à des Opus. Leur nom avait précisément été inventé par Kandinsky et Marc pour donner un titre à un recueil de textes sur l'art moderne: l'almanach "Der blaue Reiter " (1912). Ce titre qui a donné son nom au groupe référait à la fois au thème de prédilection de Marc (le cheval bleu) et à celui de Kandinsky, le cavalier, pour les intégrer dans une unité supérieure. Alors qu'un parti au nom propre et à l'appareil stables peut au fil du temps défendre des positions très hétérogènes, un groupe fondé 
sur des aspirations esthétiques est indissociable de l'identité de ses membres, il se forme à travers une conviction et un combat, et il disparait quand cet engagement perd son sens.

\section{Internet}

47 J'aimerais à présent considérer un cas sans aucun doute plus délicat: celui de productions "textuelles» qui, bien qu'elles s'inscrivent dans une démarche qui participe de la littérature, mettent en question la notion même d'opus. Par exemple, est-ce que l'auteur d'un blog peut prétendre au statut d'" auctor ", susceptible d'une image d'auteur?

Prenons l'exemple du blog de Misspastouche, tenu en 2007 par une caissière des hypermarchés Leclerc. Il a reçu un nombre considérable de visites, à l'adresse : http:// caissierenofutur.over-blog.com/. En voici un extrait :

Acte 1

Vous êtes confortablement installée derrière votre caisse sur votre chaise (Chance ! Celle-ci est un bon état et tient le choc quand vous vous adossez dessus). Vous jetez un œil distrait sur votre badge où est inscrit votre prénom, une petite maxime du style " A votre service ", « Puis-je vous aider ? ", « Le temps d'un sourire et je suis à vous » ou encore «Que puis-je faire pour vous?» et le nom de l'entreprise avec le logo. Juste à côté de votre TPE (la machine à carte bancaire), est affiché un mot avec quelques directives et explications de la direction du genre : «Pour tout paiement par chèque, merci de présenter une pièce d'identité ", le logo de la chaîne du magasin apparaît au bas de la feuille scotchée. Un coup d'œil circulaire et vous découvrez en tête de gondole des produits d'appels: des articles vantés dans le dernier prospectus (distribué dans des dizaines de milliers de boîtes aux lettres et proposé à l'accueil du magasin), les prix sont inscrits sur une pancarte flashy, le logo du magasin est visible en gros sur l'affiche. Un peu plus loin dans le rayon, vous apercevez une grande affiche (elle doit dépasser le mètre en hauteur) avec de la publicité pour un produit quelconque, le logo de l'enseigne est évidemment bien présent. Vous tournez la tête côté galerie et le nom du magasin est collé aux murs à divers points stratégiques. Bref, qu'importe l'endroit où votre regard se porte, vous voyez le nom de l'enseigne.

Ce passage constituerait une page de roman tout à fait légitime. Les conditions semblent réunies pour qu'il y ait opus : un nom d'auteur (pseudonyme), la singularité d'une expérience et d'un point de vue marqués par un style, une permanence dans le temps assurée par l'adresse, l'ajout régulier de textes nouveaux et la mise en archive des précédents. Ajoutons à cela que les réactions des internautes permettent à la blogueuse de gérer ce qu'on peut considérer comme une image d'auteur.

Il surgit cependant une difficulté : le médium, en l'occurrence Internet, n'a-t-il pas précisément pour effet de saper certaines conditions de l'accès au statut d'" auctor »?

La prolifération des blogs met en effet hors jeu une contrainte qui n'apparaissait pas clairement quand le livre ou le manuscrit dominaient: la rareté. Certes, depuis que l'imprimerie existe on n'a cessé de déplorer l'excès de livres, et donc d'auteurs, mais rien de comparable avec ce qui se passe aujourd'hui. D'autant plus qu'Internet offre au blogueur la possibilité de mettre en ligne un nombre illimité de textes, de longueurs considérables et au rythme qu'il veut. A ces textes sont en général associées des images fixes, des vidéos ou de la musique. En d'autres termes, rien ne règle plus la production, sinon le bon vouloir du blogueur et les contraintes imposées par le logiciel. 
52 C'est que l'accès à la « publication » n'y est plus limité par des intermédiaires. Dans le régime imposé par le livre, ces intermédiaires étaient de deux sortes: a) les professionnels de l'édition, qui sélectionnaient les textes et les profilaient dans des séries (en particulier les collections); leur sélection fonctionnait aussi comme une certification de qualité, tant sur le plan matériel (l'orthographe en particulier) qu'intellectuel ; b) les professionnels de l'impression : l'imprimerie était une activité liée à une technologie complexe et relativement coûteuse sur laquelle le producteur du texte, sauf exceptions, n'avait guère de prise. Outre ces deux types d'intermédiaires, le blog permet d'en supprimer un troisième : le critique. La presse fonctionnait comme un filtre puissant qui, parmi les postulants à la notoriété, ne sélectionnait qu'un nombre réduit de textes.

53 Avec Internet le statut d' " auctor » apparaît ainsi doublement menacé. La prolifération des producteurs et des énoncés rend très problématique le détachement de figures saillantes sur ce fond ; les intermédiaires s'évanouissent, au profit d'une confrontation directe entre un lecteur aléatoire et une offre infinie. Par des processus d'ordre épidémique tel ou tel blogueur peut émerger un moment de l'anonymat, mais la stabilisation d'une figure apparaît problématique. De toute façon, le tiers qui consacre un texte n'est plus une voix autorisée (un professeur, un critique), mais un essaim d'individus pseudonymes qui réagissent directement sans être eux-mêmes accrédités. Ce qui rend difficile la constitution d'une image d'auteur consistante.

L'identité même de l'œuvre devient problématique, dans la mesure où la stabilité des textes est incertaine, et avec elle la possibilité même de construire une mémoire. A la différence du texte traditionnel, dans lequel les modifications d'une édition à l'autre s'inscrivent dans un travail de "réglage » de l'opus en fonction d'une trajectoire dans l'institution littéraire, les modifications incessantes des blogs obéissent à des contraintes à très court terme ; il faut en particulier les renouveler sans cesse pour ne pas lasser les visiteurs qui en un clic peuvent se transporter ailleurs. A chaque heure, chaque minute le texte mis en ligne peut être modifié dans son contenu, sa présentation ou dans sa position dans l'architecture du site ; si bien qu'il est impossible d'affirmer quelle est la «bonne » version du texte. La prolifération des modifications finit par dissoudre l'idée même d'une transformation globale du texte. Ce phénomène est aggravé par le caractère composite des «textes » sur Internet : non seulement parce qu'on a affaire à des « hyperstructures » (Adam \& Lugrin 2000) qui déploient divers modules en tableaux, mais parce que la multimodalité y est de règle (sons, textes, images fixes et mouvantes). On ne retrouve pas la situation traditionnelle des « avanttextes ", les brouillons en particulier, qui, comme leur nom l'indique, supposent une démarcation nette entre le texte publié et ce qui est placé avant lui. Même quand l'auteur traditionnel modifiait son texte d'une édition à l'autre, il était facile de comparer les différentes versions et d'établir un apparat critique. Rien de tel avec les «textes» numériques, puisque la notion d'avant-texte n'est plus pertinente pour décrire le passage d'une « version » à une autre de ce qui est mis en ligne.

Dans ces conditions, on comprend que le blogueur qui aspire à devenir auteur soit tenté de passer au statut d'écrivain traditionnel. Le succès rencontré auprès des internautes a permis à Anna Sam, la caissière blogueuse, de publier ses textes sous le titre Les tribulations d'une caissière, paru en juin 2008 chez Stock. Ce qui a permis de découvrir qu'elle avait 29 ans, qu'elle avait un DEA de littérature et qu'elle travaillait pour payer ses études. Elle a ainsi acquis ainsi une "image d'auteur », confortée par une série de 
passages à la télévision, dans les radios et les fêtes du livre. Son blog s'est alors converti en un blog en quelque sorte "mixte", où les textes sur la vie de caissière sont associés à de multiples annonces promotionnelles sur les séances de dédicace de l'auteur et ses autres activités publiques.

On pourrait penser que cet exemple consacre in fine la domination du médium traditionnel, le livre imprimé : le blogueur finit par publier un livre. Mais les choses sont sans doute plus complexes. Dans le système traditionnel l'éditeur prenait le risque d'éditer un texte qu'il jugeait devoir plaire à un certain public; en revanche, avec Anna Sam l'édition intervient quand le public a déjà consacré le blogueur. Du même coup, le problème pour les blogueurs qui aspirent à la reconnaissance est de se détacher de la multitude. Ce qui, à n'en pas douter, pourra de moins en moins être laissé au hasard. Surtout si, comme on peut le penser, il se développe progressivement, des modes d'accès au statut d'" auctor » qui soient spécifiques au Web.

Regardons à présent un beaucoup cas bien différent, celui d'un blog pris au hasard sur Internet : le «Blog de Julie », intitulé « Enfin-Libre $»^{13}$. En voici un extrait :

Samedi 8 novembre 2008

Gueule de bois?

L'autre jour, nous avons mangé une fondue bourguignone (sic). Après le repas, ma mère a laissé l'huile refroidir sur le plan de travail. Bah... son estomac a pas trop aimé. J'ai vu le chat, dans l'entrée, sur le tapis, là où le sol chauffe. Il avait pas l'air en top forme. J'appelle ma mère, qui constate elle aussi que le père Léon est patraque. Après vérification, nous nous sommes rendu compte qu'il avait bu de l'huile... Du coup, il est resté pendant presque deux jours tout patraque, il a presque rien mangé. On a su qu'il allait mieux... quand il recommençait monter sur la table! ça ne lui a visiblement pas servi de leçon!

[http://enfin-libre.over-blog.net/article-24413205.html]

Le texte est agrémenté par une photo du chat en question:

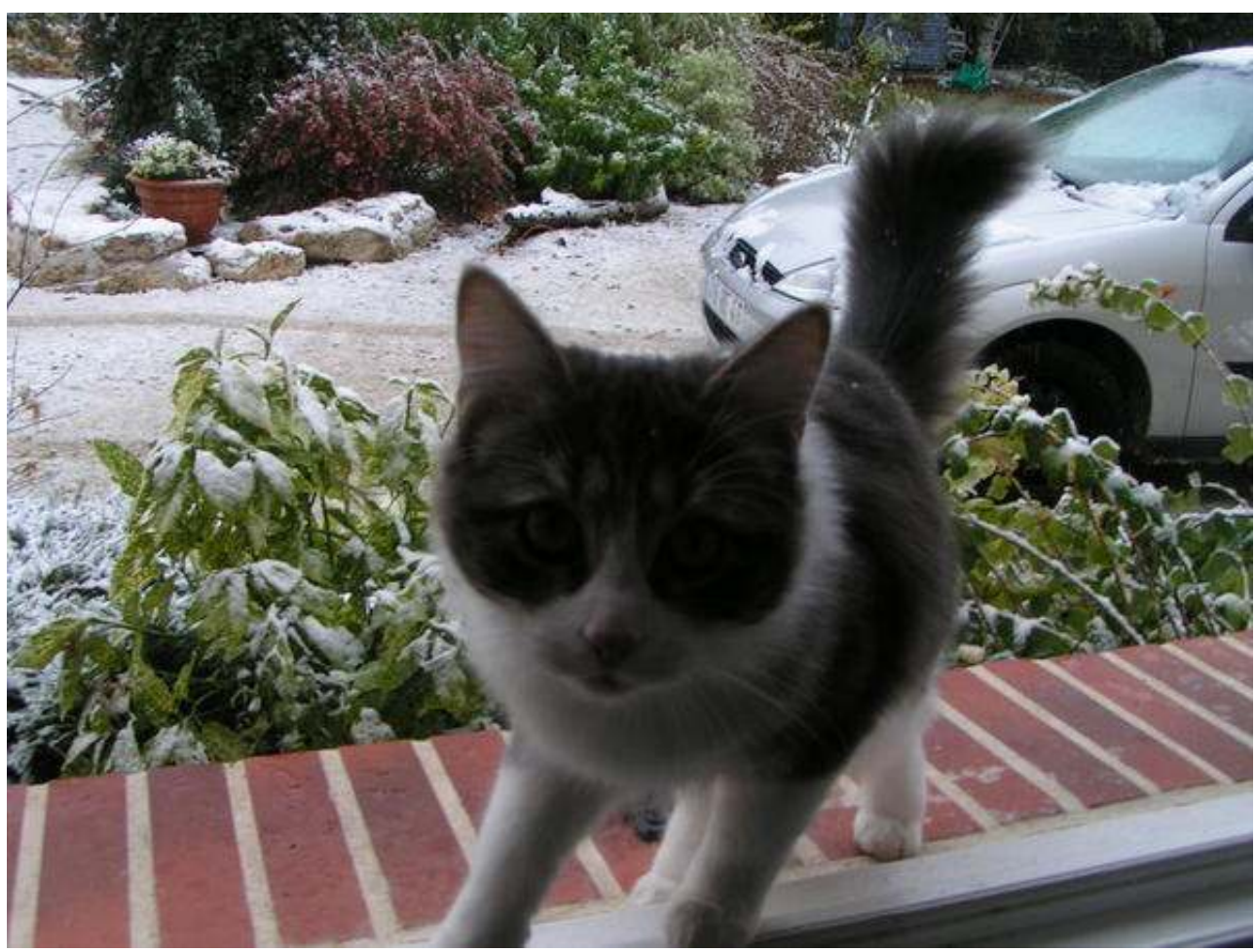



de Leclerc. Ici, on a affaire à une sorte de journal intime; avec cette différence qu'auparavant ce genre d'écrit n'était publié que si son auteur avait déjà accédé au statut d'" auctor ", associé à un Opus majeur.

Mais les choses se compliquent quand en dessous on trouve le poème suivant :

Mardi 28 octobre 2008

Like a bridge over troubled water

Quelqu'un peut-il lui dire

Que je l'aime

Quelqu'un peut-il lui dire

Que le quotidien la tue, tu sais

Quelqu'un peut-il lui dire

Qu'elle n'est pas un animal en cage.

Qu'elle a besoin d'un peu de temps

Un peu plus de temps.

Je l'aime

Et je découvre qu'aimer ça fait mal.

Quelqu'un peut-il lui dire tout ça?

Moi je n'y arrive pas.

Les seuls mots qui sortent de ma bouche sont inutiles.

Je t'aime. Tu me manque.

Et on tourne encore en rond.

Mais que peut-on y faire?

Se donner un peu de mal suffirait-il?

Voler un peu de temps.

J'ai peur.

Qu'un jour la liberté m'appelle trop fort

Me disant de m'envoler.

Mais je l'aime.

Je veux juste un peu de temps

Avec Lui

Et juste Lui.

Juste Nous.

Mais quelqu'un peut-il lui dire

Que je l'aime?

Je l'aime.

Lui...[http://enfin-libre.over-blog.net/article-24175604.html]

61 Ce texte relève-t-il du discours littéraire ? D'un certain point de vue cela ne fait aucun doute. Dès lors, on est tenté de dire que son répondant peut prétendre à devenir «auctor». En fait, on ne peut pas dire que toutes les conditions soient réunies pour convertir son auteur en acteur identifié de quelque espace littéraire. Noyé dans la série des textes du blog, lequel est lui-même noyé dans l'espace sans bords d'Internet, ce poème semble voué à rejoindre cette infinité de poèmes de circonstance qu'on a produits depuis des siècles. 

d'écriture, ne sont pas sans faire penser à l'énorme production galante du $17^{\mathrm{e}}$ siècle, dont on pourrait dire qu'il s'agit d'une littérature «sans auteurs ». Formule qui peut s'entendre de diverses manières :

- une littérature écrite par des gens qui se refusent à être considérés comme des professionnels des belles lettres ;

- une littérature de personnes qui ne publient pas leurs textes ;

- une littérature dont les producteurs, s'ils publient, ne se donnent pas pour les auteurs de ce qu'ils publient ;

- une littérature socialisée, immergée dans des interactions immédiates, dont elle ne se détache pas ;

- une littérature où il y a trop de producteurs pour qu'on puisse en distinguer un.

\section{type de production, la littérature orale :}

Après tout, la littérature orale traditionnelle était en un sens une littérature sans auteurs, un patrimoine collectif accumulé sans référence à des individus. En revanche, les nouveaux romans et poèmes, les nouvelles pièces de théâtre et les nouvelles étaient des œuvres d'artistes bien définis dont elles portaient le nom ou le pseudonyme ${ }^{14}$.

Dans le cas des blogs les producteurs sont individués. Mais c'est l'absence de contraintes de raréfaction qui fait problème. Dans le cas de la littérature orale on postule l'existence d'un nombre indéfini de producteurs-récitants antérieurs, sans qu'on puisse remonter à un texte originel stable ou à une individualité créatrice qui ne soit pas nimbée de légende, ou mythique.

On est alors incité à réserver le statut d'« auctor » aux figures que l'univers traditionnel de l'écrit - manuscrit ou imprimé - a consacrées. Pour qu'une Julie devienne auteur, dira-t-on, il suffit qu'elle rassemble ses poèmes dans quelque livre ou publie son blog sur papier, le détachant des flux anonymes d'Internet. Mais, ce faisant, on constitue en norme implicite le type d'auctorialité associé à l'écriture et à l'imprimé. Or on se trouve aujourd'hui dans une phrase de transition entre le régime traditionnel de domination de l'imprimé et un régime «numérique » en transformation perpétuelle. Il est très difficile de savoir si Internet ne va pas créer dans son propre espace de nouvelles formes d'accès au statut d' "auctor ", de nouveaux opérateurs de raréfaction et de certification des textes, qui vont coexister avec les précédentes. Un analyste du discours conséquent ne peut que prendre acte de la transformation de ces éventuelles évolutions de la figure d'auteur.

\section{BIBLIOGRAPHIE}

Adam, Jean-Michel \& Gilles Lugrin. 2000. « L'hyperstructure : un mode privilégié de présentation des événements scientifiques ", Les Carnets du CEDISCOR 6, 133-150

Argumentation et Analyse du Discours, 3 | 2009 
Amossy, Ruth (dir.). 1999. Images de soi dans le discours. La construction de l'ethos (Lausanne-Paris : Delachaux \& Niestlé)

Booth, Wayne C. 1961. The Rhetoric of Fiction (Chicago : University of Chicago Press)

Foucault, Michel. 1969a. « Qu'est ce qu'un auteur?», conférence publiée dans le Bulletin de la Société française de philosophie, 63 : 3, 73-104 [repris dans Dits et écrits I, 1954-1975. 1994 (Paris : Gallimard), 817-849]

Foucault, Michel. 1969b. L'archéologie du savoir (Paris : Gallimard)

Granger, Gilles-Gaston. 1990. Invitation à la lecture de Wittgenstein (Paris : Alinéa)

Herman Jan, Mladen Kozul \& Nathalie Kremer. 2008. Le roman véritable. Stratégies préfacielles au XVIII ${ }^{e}$ siècle (Oxford : Voltaire Foundation)

Leclerc, Gilles. 1998. Le sceau de l'œuvre (Paris : Seuil)

Maingueneau, Dominique. 1987. Nouvelles tendances en analyse du discours (Paris : Hachette)

Maingueneau Dominique. 1993. Le contexte de l'œuvre littéraire. Enonciation, écrivain, société (Paris : Dunod)

Maingueneau, Dominique. 1995. «L'énonciation philosophique comme institution discursive », Langages 119, 40-62.

Maingueneau, Dominique. 2004. Le discours littéraire (Paris : Colin)

Maingueneau, Dominique. 2006. Contre Saint Proust (Paris : Belin)

Meizoz, Jérôme. 2002. « Recherches sur la posture : Jean-Jacques Rousseau », Littérature 126, 3-17

Viala, Alain. 1993. «Eléments de sociopoétique», Viala, Alain \& Georges Molinié. Approches de la réception. Sémiostylistique et sociopoétique de Le Clézio (Paris : PUF)

\section{NOTES}

1. Il suffit de songer aux intenses débats qu'a suscités le livre de W. C. Booth (1961), qui a introduit la notion d' " auteur implicite ».

2. Ce document, issu de la Commission Biblique Pontificale a été présenté au pape Jean-Paul II par le cardinal Joseph Ratzinger au cours de l'audience du vendredi 23 avril 1993, à l'occasion de la commémoration du centenaire de l'Encyclique de Léon XIII «Providentissimus Deus » et du cinquantenaire de l'Encyclique de Pie XII « Divino afflante Spiritu ». L'italique est de moi.

3. J'utilise dans cet article ce terme peu heureux pour en éviter d'autres (auteur, écrivain, créateur...), qui sont chargés de multiples valeurs ou qui reçoivent une définition restreinte dans ma terminologie.

4. Il est d'ailleurs membre de l'Académie française.

5. Le passage (Fixot, 1994). Ce livre n'a pas eu de succès.

6. J'excepte évidemment les philosophes comme Kierkegaard ou Sartre, quand ils écrivent des romans, ou les textes publiés sous un pseudonyme par peur de la répression politique. Le seul cas qui fasse exception est celui du philosophe français Alain (1868-1951); mais, ce n'est pas un hasard, il s'est fait un nom dans le journalisme (trois mille "propos " publiés...) et s'adressait à un public large.

7. Pour dissiper toute équivoque, nous précisons qu'en tant qu'analyste du discours, nous ne souscrivons nullement à cette conception de l'auctorialité philosophique (Maingueneau 1995). Ce 
qui nous importe ici est qu'elle participe de l'idéologie spontanée de la majorité des professionnels de la philosophie.

8. On en verra quelques exemples plus bas.

9. Dans le Trésor de la Langue Française on trouve la définition suivante: "1. Personne qui se porte garante de quelqu'un ou de quelque chose. Synon. caution, garant. Louis Bonaparte incarnait le coup d'État contre la République, l'étranglement de la liberté dont l'Assemblée demeurait la gardienne; aujourd'hui, en revanche, les libertés publiques n'ont d'autre répondant et d'autre soutien que Charles de Gaulle (MAURIAC, Nouv. Bloc-Notes, 1961, p. 134). »

10. Je me souviens avoir vu à l'époque soviétique la vitrine d'une grande librairie de Berlin-Est qui ne montrait qu'un seul livre : les discours officiels du Secrétaire général du Parti communiste Tchernienko. Il s'agissait, par un acte autoritaire, de convertir en auteur de plein droit un homme politique qui n'exprimait pas de point de vue personnel. Coup de force qui s'expliquait par le statut du Parti communiste, autorité suprême qui sanctifiait en quelque sorte les paroles de ses représentants.

11. J'excepte le cas du discours de politique générale du Premier Ministre britannique qui, comme on le sait, est lu par la Reine d'Angleterre.

12. Ce groupe d'artistes (le cavalier bleu) s'est formé à partir de 1911 à Munich. Ses acteurs principaux sont Wassily Kandinsky, August Macke, Franz Marc, Paul Klee Y ont participe également Gabriele Münter, Heinrich, Campendonk, Alexej von Jawlensky

13. http://enfin-libre.over-blog.net

14. A. A. Mazrui, Collection UNESCO en ligne : Histoire générale de l'Afrique, chapitre 19. [http:// www.unesco.org/culture/africa/html_fr/chapitre819/chapitre5.htm].

\section{RÉSUMÉS}

Le développement récent, dans le champ de l'analyse du discours, d'une réflexion sur "l'auteur » et «l'image d'auteur» met en cause les distinctions traditionnelles: l'auteur n'est ni l'énonciateur du texte, ni une personne en chair et en os, qui relève du contexte. Quant à «l'image d'auteur », elle n'appartient ni au producteur du texte ni au public; elle est le produit d'une interaction entre des intervenants hétérogènes.

Tout d'abord, on s'efforce de clarifier le terme "auteur ", qui peut avoir un fonctionnement relationnel (« l'auteur de ce tract») et un fonctionnement référentiel (« un auteur important»). On distingue trois dimensions dans la notion d'auteur: 1) l'instance qui répond d'un texte: « auteur-répondant ", 2) «l'auteur-acteur », qui, organisant son existence autour de l'activité de production de textes, 3) l'auteur corrélat d'une œuvre, d'un opus: l'«auteur-auctor», qui est susceptible d'avoir une « image d'auteur».

On cherche ensuite à définir à quelles conditions une entité peut accéder au statut d'« auctor». L'existence d'une nom propre ne suffit pas. Divers cas sont étudiés : les hommes politiques, qui ne rédigent pas eux-mêmes leurs discours, les marques commerciales, les agences de publicité, les êtres collectifs (partis politiques, groupes d'artistes...).

Enfin on aborde les problèmes posé par le développement d'Internet: l'auteur d'un blog peut-il prétendre au statut d'«auctor»? Sur Internet le statut d'«auctor» apparaît menacé par la prolifération des producteurs et des textes, par la disparition des médiateurs et par l'instabilité des textes. Cette réflexion est illustrée par l'étude de deux blogs: celui d'une caissière d'hypermarché qui est devenue célèbre grâce à son blog et celui d'une blogueuse inconnue 
nommée «Julie ». Dans la phrase de transition actuelle entre le régime traditionnel où dominait l'imprimé et un régime "numérique » en transformation perpétuelle, on doit se demander si Internet va ou non créer de nouvelles formes d'auctorialité.

The recent development, in the field of Discourse Analysis, of a reflection on the notions of "author" and "image of the author" calls into question traditional distinctions: authors are neither the "enunciators" of the text nor persons in the flesh, who are part of the context. As for the "image of the author", it depends neither on the producer of the text nor on the audience: it is the result of the interaction between heterogeneous stakeholders.

To begin with, we shall try to clarify the term " author ", which can establish a relation ("the author of this pamphlet") or designate an individual ("an important author"). A distinction is made between three dimensions of the notion of author : 1) the entity that answers for a text: the author as a "guarantor", 2) the "author" as an "actor" who has a specific status in society, 3) the author as correlated to a work, an opus: the author-auctor, who can be associated with an image.

We then try to analyse under what conditions an entity can rise to the status of "auctor". Having a proper name is not sufficient. Various cases are studied: the politicians, who do not write their discourses, company's brands, advertising agencies, collective beings (political parties, groups of artists...).

Finally, we tackle the problems raised by Internet: can the author of a blog claim to be an "auctor" ? On the Web the status of auctor is at risk, because of the proliferation of producers and texts, the elimination of the mediators and the instability of texts. This reflection is illuminated by studying two blogs: that of a supermarket cashier who got famous thanks to her blog and that of an unknown woman named "Julie". In the current transition from the traditional regime of textuality, when printing prevailed, and a digital regime which is constantly evolving, one must wonder whether Internet will or will not create new forms of authorship.

\section{INDEX}

Mots-clés : analyse du discours, auctor, auctorialité, auteur, blog, image d'auteur, Internet, répondant

Keywords : auctor, author, authorship, blog, discourse analysis, guarantor, image of the author, Internet

\section{AUTEUR}

\section{DOMINIQUE MAINGUENEAU}

Université Paris 12, Céditec, Institut Universitaire de France 